July 2007

\title{
Plant-derived EpCAM antigen induces protective anti-cancer response.
}

\author{
Robert Brodzik \\ Sergei Spitsin \\ Max Golovkin \\ Katarzyna Bandurska \\ Carla Portocarrero
}

See next page for additional authors

Follow this and additional works at: https://jdc.jefferson.edu/cbfp

Part of the Amino Acids, Peptides, and Proteins Commons

Let us know how access to this document benefits you

\section{Recommended Citation}

Brodzik, Robert; Spitsin, Sergei; Golovkin, Max; Bandurska, Katarzyna; Portocarrero, Carla; Okulicz, Monika; Steplewski, Zenon; and Koprowski, Hilary, "Plant-derived EpCAM antigen induces protective anti-cancer response." (2007). Department of Cancer Biology Faculty Papers. Paper 15.

https://jdc.jefferson.edu/cbfp/15

This Article is brought to you for free and open access by the Jefferson Digital Commons. The Jefferson Digital Commons is a service of Thomas Jefferson University's Center for Teaching and Learning (CTL). The Commons is a showcase for Jefferson books and journals, peer-reviewed scholarly publications, unique historical collections from the University archives, and teaching tools. The Jefferson Digital Commons allows researchers and interested readers anywhere in the world to learn about and keep up to date with Jefferson scholarship. This article has been accepted for inclusion in Department of Cancer Biology Faculty Papers by an authorized administrator of the Jefferson Digital Commons. For more information, please contact: JeffersonDigitalCommons@jefferson.edu. 


\section{Authors}

Robert Brodzik, Sergei Spitsin, Max Golovkin, Katarzyna Bandurska, Carla Portocarrero, Monika Okulicz, Zenon Steplewski, and Hilary Koprowski 


\title{
Plant-derived EpCAM antigen induces protective anti-cancer response
}

\author{
Robert Brodzik, Sergei Spitsin, Max Golovkin, Katarzyna \\ Bandurska, Carla Portocarrero, Monika Okulicz, Zenon Steplewski,

\section{Hilary Koprowski}

Biotechnology Foundation Laboratories,

Thomas Jefferson University, 1020 Locust Street, Philadelphia, PA 19107, USA

e-mail: h_koprowski@jefferson.edu;

hilary.koprowski@jefferson.edu

Present Address:

M. Okulicz

Department of Animal Physiology and Biochemistry, August Cieszkowski Agricultural University, Wolynska 35, 60-637 Poznan, Poland

\section{Abstract}

Immunotherapy holds great promise for treatment of infectious and malignant diseases and might help to prevent the occurrence and recurrence of cancer. We produced a plant-derived tumor-associated colorectal cancer antigen EpCAM (pGA733) at high yields using two modern plant expression systems. The full antigenic domain of EpCAM was efficiently purified to confirm its antigenic and immunogenic properties as compared to those of the antigen expressed in the baculovirus system (bGA733). Recombinant plant-derived antigen 
induced a humoral immune response in BALB/c mice. Sera from those mice efficiently inhibited the growth of SW948 colorectal carcinoma cells xenografted in nude mice, as compared to the EpCAM-specific mAb CO171A. Our results support the feasibility of producing anti-cancer recombinant vaccines using plant expression systems.

\section{Keywords}

Plant biotechnology, Colorectal cancer, Recombinant subunit vaccine

\section{Abbreviations}

TAA

Tumor-associated antigen

EpCAM/GA733 Colorectal cancer-associated antigen

bGA733 Baculovirus-derived GA733-2

pGA733 Plant-derived GA733-2

mAb Monoclonal antibodies

\section{Introduction}

The concept of cancer vaccination as a preventive measure is of increasing interest to oncology in view of advances in modern molecular biology and biotechnology $[7,14,15]$. Several anti-cancer vaccines for the treatment of minimal residual disease are currently in human clinical 
trials $[6,21]$. The $\mathrm{T}$ - and $\mathrm{B}$-cell immune responses against tumorassociated antigens (TAA) could lead to efficient elimination of tumor cells without harming the surrounding normal tissue, as well as help to generate long-lasting immunological memory against tumor recurrences, as already shown in humans and animals [8]. There is also strong evidence supporting the use of recombinant antigen vaccines, either alone, or in combination with hormonal, chemotherapeutic and monoclonal antibody approaches, to treat colorectal cancer $[1,5,19,22$, 26].

The use of plant biotechnology to produce pharmaceutical and industrial proteins has clear economic scalability and safety advantages as compared to traditional microbial and mammalian production systems [9, $11,12,16,17]$. Plants offer potentially very high expression level of recombinant proteins $[10,11]$. However, the main advantage of plantbased expression systems is lack of harmful or even lethal contaminants (viruses, toxins, prions, oncogenes) that might contaminate other production systems $[2,9-12]$. Current efforts are focused on maximizing expression levels and accumulation of functional plant-produced proteins, preferably in soluble form to facilitate purification $[4,5,10,11,13,23]$. We previously expressed the GA733-2 antigen in planta using a tobacco mosaic virus-based system. Although, mice immunized with the plantproduced product demonstrated an antigen-specific immune response, 
small amounts of plant-derived material precluded complete immunological assessment [26].

The use of modern transient and stable plant transformation systems now allows the high yield production of GA733-2 TAA in plants. We found that fusion to the endoplasmic reticulum (ER) retaining signal led to increased levels of protein accumulation in plant cells, and an efficient immuno-affinity purification procedure yielded EpCAM antigen (pGA733) in sufficient amounts to confirm its protective immunogenicity in mice. Antigen-specific serum antibodies from these mice inhibited tumor cell growth in xenografted nude mice. Our results confirm the utility of plants in high-yield and inexpensive production of drugs for cancer immunotherapy.

\section{Materials and methods}

EpCAM expression cassette design

A DNA fragment of GA733-2 antigen extracellular domain (aa 24-264) $[20,25]$ was amplified by the following primers: $\mathrm{F}-5^{\prime}-$ CCATGGCTCAGGAAGAATGTGTCTGT-3' and R-5' GTCGACTTTATCATCATCATCAAGATCTITTAG ACCCTGCATTGAGAATTCA3', which carry NcoI and BglII sites, respectively. The product was cloned into the pGEMT vector (Promega, Madison, WI, USA), sequenceverified, and recloned into plasmid pBIV-1.3Tag (Plant Research 
International, Wageningen, NL, USA) comprised of the RbcS1 promoter, C-terminal tags (c-myc and $\mathrm{His}_{6}$ ) and the KDEL retention ER signal. The resulting expression cassette was transferred (AscI/PacI) into the plant binary vector $p B I N-$ Plus (Plant Research International), resulting in plasmid $p R B 74$, which was placed into Agrobacterium tumefaciens strain LBA4404 for stable plant transformation. The GA733 NcoI/SacI fragment from the $p B I V$-based construct was recloned into the carrier pro-vector plasmid pICH11599 (Icon Genetics, Haale (Saale), Germany) and placed in Agro-strain GV3101 for vacuum-infiltration "magnifection" of wild type Swiss chard plants.

Transient and constitutive plant transformation

For rapid production of recombinant antigen, we used the magnifection procedure $[10,11,13,18]$. Agro-cultures carrying the expression cassette were mixed with cultures carrying pre-manufactured helper plasmids ( $p I C H 14011$ and $p I C H 17620)$ (Icon Genetics) and applied to mature (6- 8 weeks old) Beta vulgaris var. cicla (Swiss chard) plants. Plant tissues were harvested after 7-10 days and analyzed by Western blot and ELISA. Low-alkaloid Nicotiana tabacum cv. LAMD609 (tobacco) (Oxford Tobacco Research Station, Oxford, NC) was used for leaf explants agro-mediated transformation [5] with $p R B 74$. Independent transgenic lines were selected in medium containing kanamycin $(100 \mathrm{mg} / \mathrm{l})$ and used 
for molecular characterization.

Isolation and purification of plant ( $p$ ) GA733 protein

Plant leaf tissues were collected and processed as described $[5,13]$. After centrifugation, supernatants were additionally successively clarified through a Miracloth (Calbiochem, La Jolla, CA, USA) and a $0.45-\mu \mathrm{m}$ filter (Millipore, Bedford, MA, USA). Soluble protein extract was applied on a murine mAb Ab733-immobilized HighTrap column (Amersham, Piscataway, NJ, USA) or purified using $\mathrm{His}_{6}$ and c-myc tags as described [13]. Eluates of recombinant pGA733 protein were combined, dialyzed against PBS buffer and brought to a final concentration $1 \mathrm{mg} / \mathrm{ml}$ using Amicon Ultra spin-column with a 10-kDa cut-off (Millipore). Aliquots were frozen in liquid nitrogen and stored at $80^{\circ} \mathrm{C}$. For analysis extracts were resolved by SDS-polyacrylamide gel electrophoresis (PAGE) and either stained or transferred to a nitrocellulose membrane (Bio-rad, Hercules, CA, USA), blocked with $5 \%$ non-fat milk and incubated with murine c-myc mAb (Calbiochem, San Diego, CA, USA) or mAb Ab733 followed by secondary anti-mouse Fc-specific Ab conjugated to horseradish peroxidase (Sigma, St Louis, MO, USA) diluted 1:10,000. Reactive protein bands were visualized using chemiluminescent substrate for peroxidase (Pierce, Rockford, IL, USA). 
Immunological analysis of pGA733 in mice

Eight-week-old female BALB/c mice (five per group) were injected with three doses $(5 \mu \mathrm{g})$ of pGA733 in a total volume of $100 \mu \mathrm{l}$ at 2-week intervals. First and second immunizations were given subcutaneously (s.c.) with complete and incomplete Freund's adjuvant (Difco, Detroit, MI, USA), respectively; the third dose was administered inter-peritoneally (i.p.) in saline. Control groups received $5 \mu \mathrm{g}$ per injection of baculovirusproduced bGA733 (kindly provided by Dr William Wunner, Wistar Institute, Philadelphia, PA, USA) or total soluble protein extract (TSP) from non-transgenic wild-type plants. Blood samples were collected by retro-orbital bleeding before experiment and 10 days after the second immunization; 10 days after the third immunization, mice were sacrificed and bled by cardiac puncture. Sera were analyzed by ELISA and Western blotting. ELISA for bGA733 ( $1 \mu \mathrm{g} / \mathrm{ml}$ in PBS) was performed essentially as described [23]. Antigen-specific antibodies were detected using goat antimouse IgG (BD Biosciences, San Jose, CA, USA), goat anti-mouse IgG2b and IgG3 (Bethyl Labs, Montgomery, TX, USA) and rat anti-mouse IgG1 and IgG2a (BD Biosciences). Results are presented as mean \pm SD.

Inhibition of tumor growth in vivo

Human colorectal carcinoma cells SW948 were maintained according to 
the supplier's instructions (ATCC) in DMEM supplemented with $10 \%$ FBS. Six-to-8-week-old BALB/c nu/nu mice (5 per group) (Charles River Laboratories, Wilmington, MA, USA) were inoculated s.c. with $10^{6}$ cells on the back of the neck followed immediately by four i.p. injections at 3-day intervals with $100 \mu \mathrm{l}$ of serum from pGA733 or bGA733 immunized BALB/c mice for a total of $400 \mu$ during 7 days. Control groups were injected with $100 \mu \mathrm{l}$ of TSP serum or $100 \mu \mathrm{g}$ of mAb CO17-1A (Centocor, Horsham, PA, USA) using the same regimen. Tumor growth was recorded at $10,15,17,19,22,24,26,29,31,35$ and 38 days after initial injection and calculated based on the three major diameters measured with graduated calipers. At the end of the experiment, mice were euthanized by $\mathrm{CO}_{2}$ inhalation in accordance with the institutional guidelines for animal welfare.

\section{Results}

Production and puriWcation of pGA733 (EpCAM)

cDNA of the extracellular domain of antigen GA733 (aa 24-264) was inserted into the $p B I V-1.3 T a g$ vector as a translational fusion with the ER signal peptide; c-myc and $\mathrm{His}_{6}$ tag epitopes and the ER retention signal KDEL (Plant Research International) were attached to the GA733 C-terminus. The antigen expression cassette containing promoter and 
termination signal was placed in the pBINPlus binary vector, yielding the construct $p R B 74$ (Fig. 1a). EpCAM coding sequence (except ER signal peptide) was subcloned into the magnICON Icon Genetics' plasmid pICH115999 (Fig. 1a) and used for transient expression of pGA733 [11, $13,18]$.

The magnifection technique provided a robust and rapid way to express recombinant pGA733 transiently in a large biomass plant, such as Swiss chard (Fig. 1b, left). The recombinant protein was readily detected by Western blotting at 7-9 days, post-infection in transfected leaf tissues (Fig. 1b, right).

Several stably transformed transgenic tobacco lines selected based on kanamycin resistance (Fig. 1c, left) showed detectable amounts of pGA733 in leaf extracts on Western blot analysis (Fig. 1c, right). Quantitative ELISA indicated a variable level of recombinant antigen expression with up to $10 \mathrm{mg} / \mathrm{kg}$ of fresh leaves (not shown). The transgenic line with the highest detectable antigen amount was selfcrossed to obtain a homozygous line.

The GA733 protein remained stable in fresh and lyophilized plant tissues at ambient temperatures or at $4^{\circ} \mathrm{C}$ and was used to obtain standardized samples at purity suitable for immunization (Fig. 1d). Plant-derived antigen was purified from tobacco and Swiss chard plants by single step antigen-specific immunoaffinity chromatography and/or by two-step 
affinity tag-specific technique, yielding $\sim 5 \mathrm{mg}$ of pGA733 from $1 \mathrm{~kg}$ of fresh leaf tissue as verified by SDSPAGE (Fig. 1d, left) and quantitative ELISA. Equal amounts of plant and baculovirus produced GA733, as determined by protein assay, produced bands of similar intensity on coomassie gel and western blot and the same OD in quantitative ELISA with monoclonal antibodies against GA733. Purified product resolved as a double-band (both $\sim 30 \mathrm{kDa}$ ) with a few additional minor bands confirmed to be subproducts of pGA733 by immunostaining analysis (right). The purity of plant-derived antigen as visualized on SDS-PAGE is $\sim 60 \%$ (left). Note that migration of the plant-derived protein was slower than that of bGA733 (Fig. 1d), possibly reXecting the presence of the cmyc and $\mathrm{His}_{6}$ tags and the KDEL peptide.

Immune response of mice injected with pGA733

All mice injected with pGA733 or bGA733 antigen mounted a strong GA733-speciWc serum antibody response, with comparable titers after either immunization as tested in ELISA against plant- and baculovirusderived antigen (Fig. 2a). As expected, both IgG1 and IgG2a/b subclasses were present in the sera, with slight predominance of IgG1 (Fig. 2b). Western blotting confirmed the serum specificity of anti-GA733 antibodies in mice immunized with bGA733 or pGA733 (Fig. 2c).

Suppression of tumor growth in mice by plant-derived vaccine 
In nude mice xenografted with SW948 colorectal cancer cells and treated with plant TSP serum, the first signs of tumor appeared 10 days after injection; thereafter, tumors grew rapidly, averaging $1,357 \mathrm{~mm}^{3}$ by day 38 (Fig. 3). The first signs of tumor appeared by day 15 in mice treated with pGA733 and bGA733 sera and by day 22 in mice treated with mAb C017-1A (Fig. 3a). In bGA733-treated group, all mice developed tumors, although development was significantly delayed in two mice. In the mAb C017-1A treated group, two mice remained tumor-free for the 38-day observation period. At 38 days, mean tumor volumes of both pGA733 and bGA733-treated mice were significantly lower than that of the TSP control group $(P<0.0001)$ (Fig. 3a, b). One mouse in pGA733 group remained tumor free throughout the 38-day observation period. In control plant TSP sera-treated group, all mice developed tumors (Fig. 3b, bottom).

\section{Discussion}

Several TAAs have been identified and their immune interactions in patients have been well characterized. In numerous cases, immune approaches to the treatment of cancer have entered the clinical stage [6, 21]. The EpCAM TAA, which has been studied for more than 20 years, is one of the best-described candidates for active immunotherapy of colorectal cancer [20]. 
In the last 2 decades, the use of plants for production of a range of different therapeutics has been explored $[9,12,16,17]$. Our recent comparison of the immune response induced by GA733 produced in baculovirus-infected insect cell culture versus GA733 produced in planta using a viral vector [26] revealed a similar antibody response to both antigens in mice, with similar tumor cell specificity in in vitro assay. However, the low expression level in this prevented comparison of the anti-cancer activity of the mouse in vivo.

In this study, we used modern plant production schemes $[5,13]$ to efficiently express the EpCAM TAA. High-yield production was achieved using the "ImpactVector system", which is using the strong RbcS1 promoter from Chrysanthemum and allows direction of antigen expression into specific plant cell compartments. Fusion of c-myc and $\mathrm{His}_{6}$ tags, as well as the ER retention signals KDEL to the C-terminus of GA733 facilitated detection and purification of the antigen. Furthermore, using this expression cassette, we produced considerable amounts of pGA733expressing leaf biomass using rapid and robust magnification of the vegetable plant Swiss chard. Recombinant antigen was expressed at levels of $5-10 \mathrm{mg} / \mathrm{kg}$ of fresh leaf material.

The recombinant pGA733 was readily extracted from the pooled leaf material in soluble form. Transgenic $T_{0}$ tobacco plants with the highest expression level were processed in a one-step, immunoaffinity-based 
purification procedure that resulted in $\sim 50 \%$ recovery of recombinant antigen from plant tissue $(\sim 5 \mathrm{mg} / \mathrm{kg})$. Although transient expression and double-step purification was even more efficient, stably transformed lowalkaloid tobacco appears to be more economically feasible.

The immune response to pGA733 appeared to be the same or only slightly weaker than that of an equal dose of bGA733 in BALB/c mice. It seems unlikely that differential reactivity, e.g., due to different glycosylation patterns in pGA733 and bGA733, underlies this observation, since similar results were obtained in ELISA with either antigen. Moreover, Western blot analysis of mouse sera against pGA733 and bGA733 at 1:10,000 dilutions revealed similar patterns, indicating high purity of both antigens. Reactivity of anti-pGA733 sera appeared to be in this case a bit higher with pGA733 compared to bGA733 (Fig. 2c). In earlier analyses of the immunological properties of pGA733 using QS21 as adjuvant, we observed a predominantly IgG1 response [26], whereas in the present study we detected a higher percentage of IgG2a/b antibodies in sera of mice immunized with either pGA733 or bGA733, although IgG1 antibodies remained a prominent subclass (Fig. 2b). This is likely due to the use of Freund's adjuvant.

Sera from mice immunized with pGA733, bGA733 or mAb CO17-1A demonstrated significant inhibition of tumor growth in comparison to TSP control sera in xenografted nude mice. There were no significant 
differences between sera from mice immunized with pGA733 or bGA733 in tumor growth inhibition in vivo. The mAb CO17-1A sera had some advantage over pGA733 and bGA733 sera during the early period (days 15-19) but the difference became insignificant later on (days 22-38) (Fig. 3a).

The possibility that pre-assembled antigen-antibody immune complexes might have superior immunogenicity [3] awaits analysis using plantderived mAb CO17-1A [15] and plant-derived EpCAM antigen.

Our data clearly demonstrate that pGA733-generated sera display comparable in vitro and in vivo activity to the sera generated against the bGA733 antigen and can efficiently inhibit tumor growth in vivo. This work points to the promise of transgenic plants as an excellent source of anticancer vaccines, particularly in the case of this attractive target-EpCAM antigen, which is known to be immunogenic in humans [24].

\section{Acknowledgments}

We thank TJU and KCC research and animal facilities for their support; G. Golovin for greenhouse work and Dr William Wunner for providing bGA733 antigen. This work was supported by a grant from Commonwealth of Pennsylvania Department of Health to Biotechnology Foundation Laboratories (H.K.) and a grant from USDA to Biotechnology Foundation Laboratories (H.K.). 


\section{References}

1 Armstrong A, Eck SL (2003) EpCAM: a new therapeutic target for an old cancer antigen. Cancer Biol Ther 2:320-326

2 Boehm R (2007) Bioproduction of therapeutic proteins in the 21st century and the role of plants and plant cells as production platforms. Ann N Y Acad Sci 1102:121-134

3 Brady LJ (2005) Antibody-mediated immunomodulation: a strategy to improve host responses against microbial antigens. Infect Immune 73:671-678

4 Brodzik R, Bandurska K, Deka D, Golovkin M, Koprowski H (2005) Advances in alfalfa mosaic virus-mediated expression of anthrax antigen in planta. Biochem Biophys Res Commun 338(2):717-722

5 Brodzik R, Glogowska M, Bandurska K et al (2006) Plant-derived anti-Lewis $Y$ mAb exhibits biological activities for efficient immunotherapy against human cancer cells. Proc Natl Acad Sci USA $103: 8804-8809$

6 Choudhury A, Mosolits S, Kokhaei P et al (2006) Clinical results of vaccine therapy for cancer: learning from history for improving the future. Adv Cancer Res 95:147-202

7 Dalgleish AG, Whelan MA (2006) Cancer vaccines as a therapeutic modality: the long trek. Cancer Immunol Immunother 55:10251032 
8 Emens LA (2006) Roadmap to a better therapeutic tumor vaccine. Int Rev Immunol 25:415-443

9 Fox JL (2006) Turning plants into protein factories. Nat Biotechnol 24:1191-1193

10 Gleba Y, Klimyuk V, Marillonnet S (2005) Magnifection-a new platform for expressing recombinant vaccines in plants. Vaccine $23: 2042-2048$

11 Gleba Y, Klimyuk V, Marillonnet S (2007) Viral vectors for the expression of proteins in plants. Curr Opin Biotechnol 18(2):134141

12 Goldstein DA, Thomas JA (2004) Biopharmaceuticals derived from genetically modified plants. Q J Med 97:705-716

13 Golovkin M, Spitsin S, Andrianov V et al (2007) Smallpox subunit vaccine produced in planta confers protection in mice. Proc Natl Acad Sci USA 104(16):6864-6869

14 Hodge JW, Greiner JW, Tsang KY et al (2006) Costimulatory molecules as adjuvants for immunotherapy. Front Biosci 11:788-803

15 Ko K, Steplewski Z, Glogowska M, Koprowski H (2005) Inhibition of tumor growth by plant-derived mAb. Proc Natl Acad Sci USA $19: 7026-7030$

16 Koprowski H (2005) Vaccines and sera through plant biotechnology. Vaccine 23:1757-1763 
17 Ma JK-C, Chikwamba R, Sparrow P, Fischer R, Mahoney R, Twyman RM (2005) Plant-derived pharmaceuticals-the road forward. Trends in Plant Sci 10:580-585

18 Marillonnet S, Giritch A, Gils M, Kandzia R, Klimyuk V, Gleba Y (2004) In planta engineering of viral RNA replicons: efficient assembly by recombination of DNA modules delivered by Agrobacterium. Proc Natl Acad Sci USA 101:6852-6857

19 Mosolits S, Markovic K, Frodin JE, Virving L et al (2004) Vaccination with Ep-CAM protein or anti-idiotypic antibody induces Th1-biased response against MHC class I- and II-restricted Ep-CAM epitopes in colorectal carcinoma patients. Clin Cancer Res 10:5391-5402

20 Mosolits S, Nilsson B, Mellstedt H (2005) Towards therapeutic vaccines for colorectal carcinoma: a review of clinical trials. Expert Rev Vaccines 4:329-350

21 Nagorsen D, Thiel E (2006) Clinical and immunologic responses to active specific cancer vaccines in human colorectal cancer. Clin Cancer Res 12:3064-3069

22 Neidhart J, Allen KO, Barlow DL et al (2004) Immunization of colorectal cancer patients with recombinant baculovirus-derived KSA (Ep-CAM) formulated with monophosphoryl lipid $A$ in liposomal emulsion, with and without granulocyte-macrophage colonystimulating factor. Vaccine 22:773-780 
23 Pogrebnyak N, Golovkin M, Andrianov V et al (2005) Severe acute respiratory syndrome (SARS) S protein production in plants: development of recombinant vaccine. Proc Natl Acad Sci USA 102:9062-9067

24 Staib L, Birebent B, Somasundaram R et al (2001) Immunogenicity of recombinant GA733-2E antigen (CO17-1A, EGP, KS1-4, KSA, EpCAM) in gastro-intestinal carcinoma patients. Int J Cancer 92:79-87

25 Szala S, Froehlich M, Scollon M et al (1990) Molecular cloning of cDNA for the carcinoma-associated antigen GA733-2. Proc Natl Acad Sci USA 87:3542-3546

26 Verch T, Hooper DC, Kiyatkin A, Steplewski Z, Koprowski H (2004) Immunization with a plant-produced colorectal cancer antigen. Cancer Immunol Immunother 53:92-99 


\section{Figures}
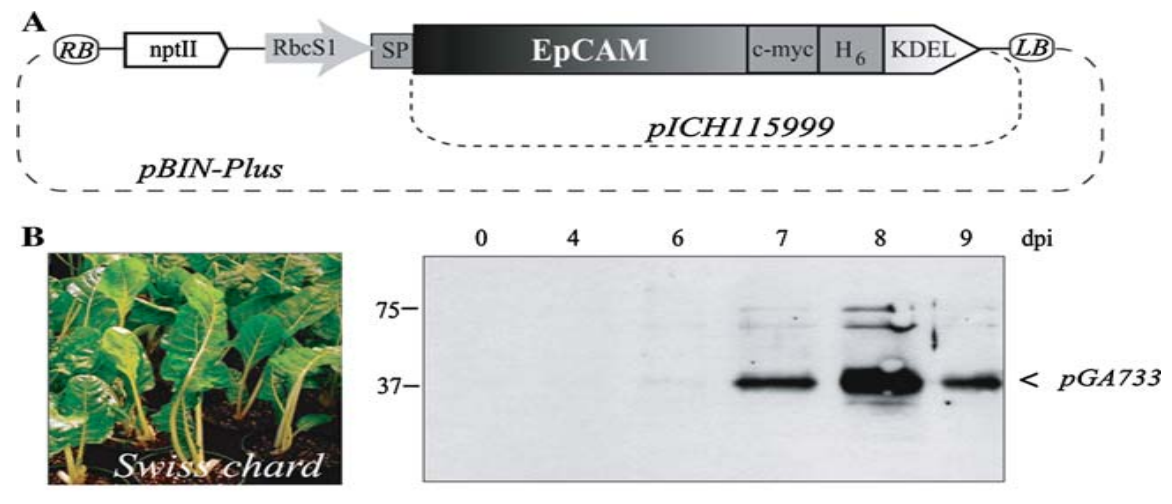

$\mathbf{C}$
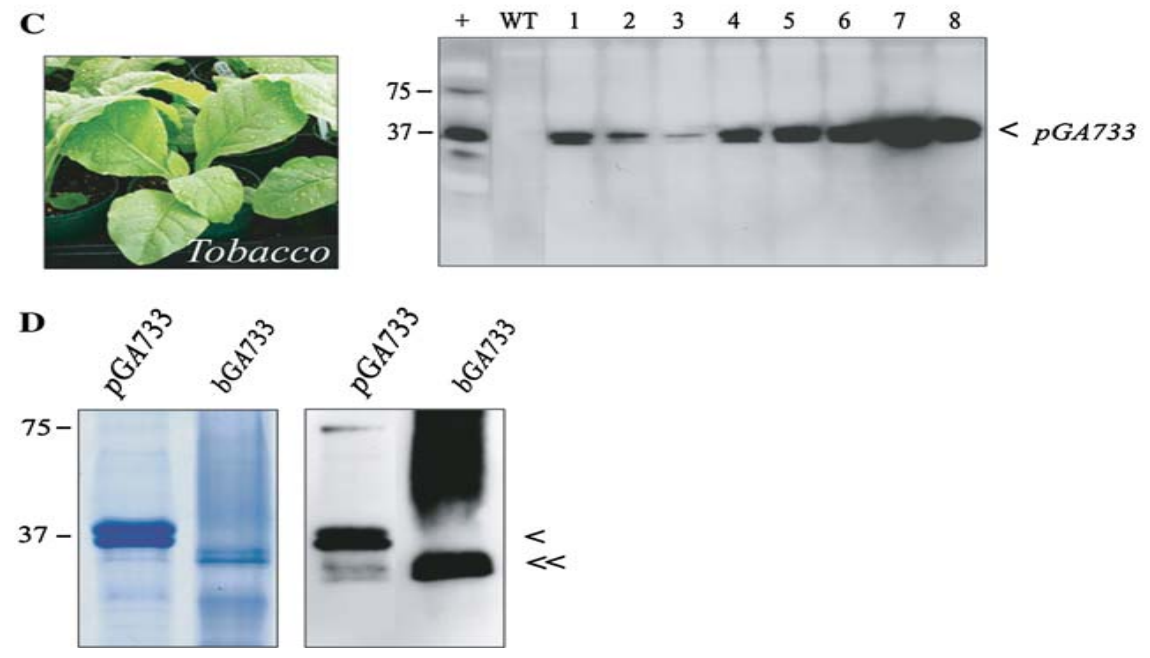

Fig. 1 Expression of EpCAM in plants.

a Colorectal cancer-associated antigen EpCAM/GA733-2 was assembled in plant expression vectors pBIN-plus (ImpactVector) and pICH115999 (MagnICON) for stable and transient plant transformation, respectively. The final expression cassette contains: the rubisco small subunit promoter, RbcS1, driving the coding sequence of EpCAM antigen; the ER signal peptide $(S P)$ and retention signal KDEL; a cassette (nptII) conferring resistance to the antibiotic kanamycin, and c-myc and $H_{6}$ tags. 
All components are located within the left $(L B)$ and right $(R B)$ borders of TDNA (plasmid $p R B 74$ ) for stable transformation.

b Six-week-old Swiss chard plants were used for MagnICON-based expression (left). Soluble $p G A 733$ was detected at 7, 8 and 9 days post inoculation ( $d p i)$ by Western blotting with antigen-specific mAb in total soluble protein (TSP) extracts (right).

c Stably transformed tobacco plants (left) and Western blotting (right) of total protein extract from this plants probed with murine mAb AB733. Positive control (+) is the bacteria-expressed GA733 antigen; protein extract from non-transgenic wild-type (wt) tobacco served as a negative control.

d SDS-PAGE and Western blotting (at 1:1000 dilution) of soluble pGA733 affinity-purified from plant leaf tissues compared with purified $b G A 733$. Numbers on the left indicate molecular size (kDa). 

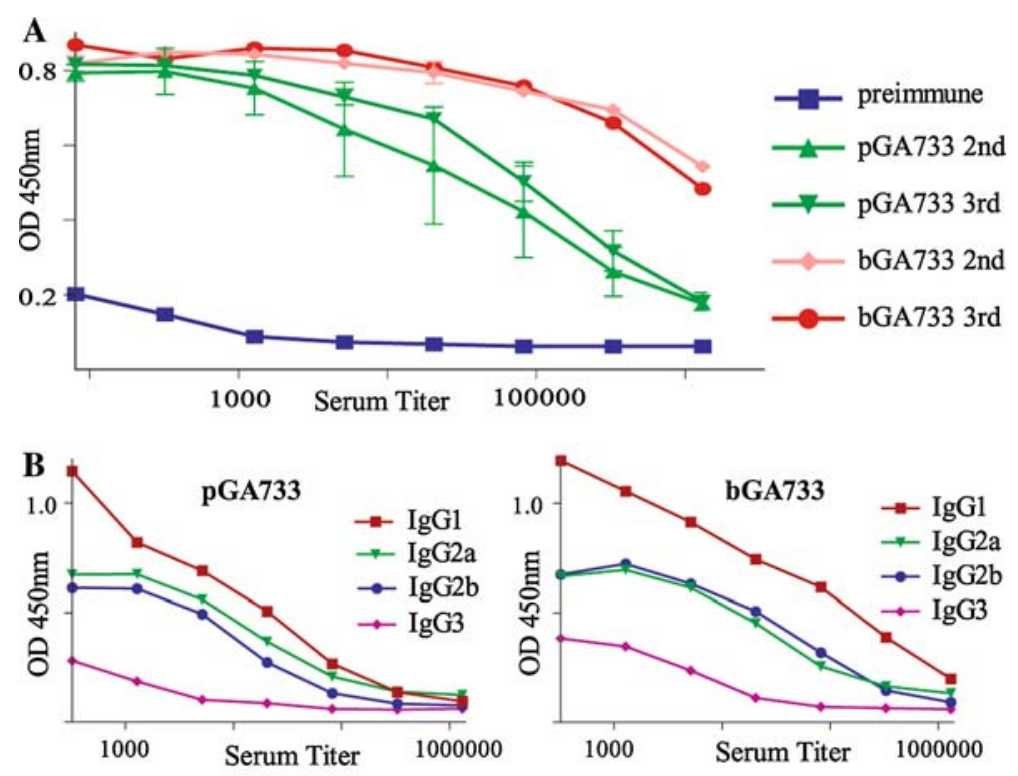

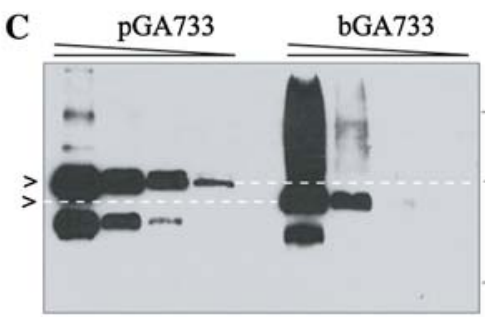

pGA733 sera (3rd)

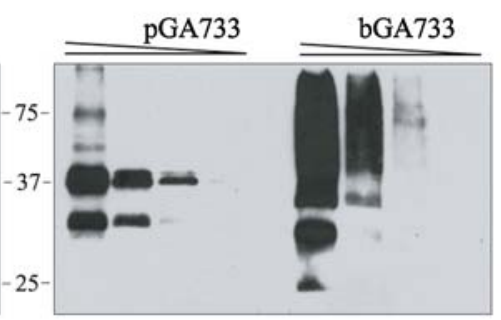

bGA733 sera (3rd)

Fig. 2 Serum antibody response to EpCAM in BALB/c mice.

a ELISA titers of sera from BALB/c mice immunized with pGA733 or bGA733 antigen. Results are presented as mean \pm SD.

b ELISA IgG subclass titers of the pooled pGA733 and bGA733 sera in panel a.

c Serial dilutions of pGA733 and bGA733 protein preparations (1:3) probed with the corresponding pooled sera (1:10,000 dilution) obtained after the third immunization of mice in panel $A$. 

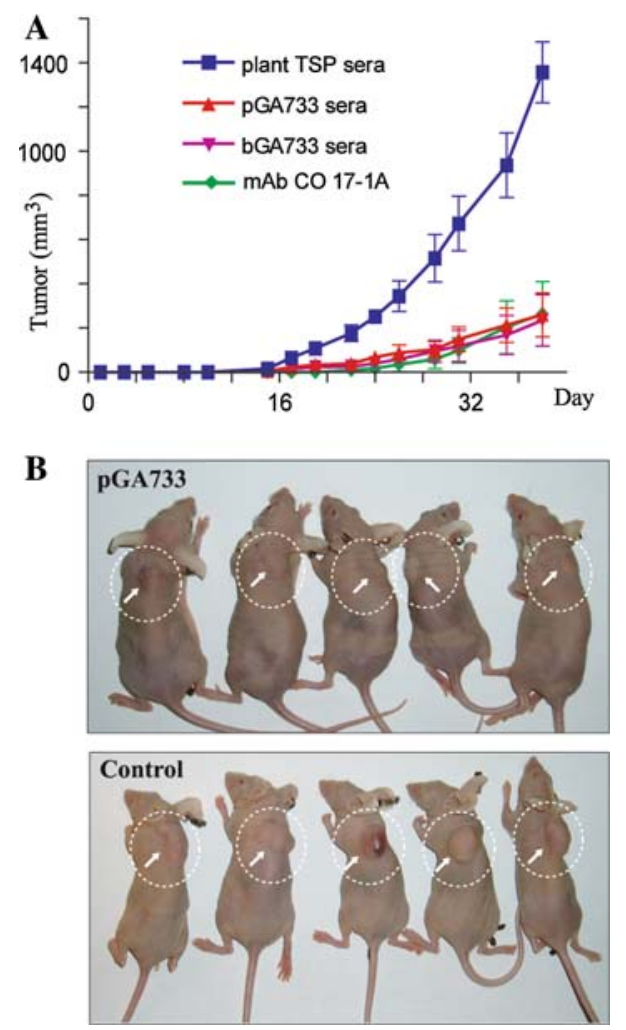

Fig. 3 Suppression of tumor growth in nude mice by pGA733-specific antibodies.

a BALB/C nu/nu mice xenografted with $10^{6}$ SW948 colorectal cancer cells were injected with serum from BALB/c mice immunized with either pGA733 or bGA733 antigen, or received serum from mock-immunized (plant TSP) mice, or murine mAb CO17-1A. At days 2, 4 and 7, all mice were injected with three additional doses of sera or antibodies. Tumor volumes $\left(\mathrm{mm}^{3}\right)$ were recorded at 10, 15, 17, 19, 22, 24, 26, 29, 31, 35 
and 38 days after initial inoculation with cancer cells. Data are given as mean $\pm S D$.

b Pictures of mice treated with pGA733 sera (top) or control plant TSP sera (bottom) were taken on day 35 after injection of tumor cells. Tumor areas are circled; arrows indicate tumor cell injection sites. 